\title{
A Measurement of the Diffuse Astrophysical Muon Neutrino Flux Using Eight Years of IceCube Data
}

\author{
The IceCube Collaboration \\ $\dagger$ http://icecube.wisc.edu/collaboration/authors/icrc17_icecube \\ E-mail: haack@physik.rwth-aachen.de, \\ wiebusch@physik.rwth-aachen. de
}

\begin{abstract}
Recently the IceCube collaboration confirmed the astrophysical neutrino flux in the muon neutrino channel with high significance using six years of data. This analysis used a likelihood approach with reconstructed muon energy and zenith angle as observables to measure the properties of the astrophysical muon neutrino flux. Additionally constraints on the prompt atmospheric neutrino flux from the decay of charmed mesons were obtained. In this contribution we will present an update to this analysis using eight years of data collected from 2009 through 2017, containing about 500,000 muon neutrino candidates with a negligible contribution of atmospheric muons.
\end{abstract}

Corresponding authors: C. Haack ${ }^{* 1}, \mathrm{C}$. Wiebusch ${ }^{1}$

${ }^{1}$ III. Physikalisches Institut, RWTH Aachen University

35th International Cosmic Ray Conference (ICRC2017)

10-20 July, 2017

Bexco, Busan, Korea

* Speaker. 


\section{Introduction}

The detection and use of high-energy astrophysical neutrinos as cosmic messengers has been an outstanding goal in the field of astroparticle physics. In 2013 the IceCube neutrino observatory [1] reported the first evidence for the existence of a diffuse flux of extraterrestrial neutrinos at high energies [2,3]. The discovery was based on neutrinos of all flavors above a few $10 \mathrm{TeV}$ that interact within the detector volume (starting events). Shortly after, this signal was confirmed by a complementary measurement utilizing through-going and starting muons from charged-current muon neutrino nucleon interactions close to the detector volume [4, 5]. Both measurements showed a clear excess of high-energy events, that cannot be explained by atmospheric neutrino or muon backgrounds.

The most recent published analysis of through-going muons [5] is based on six years of data, collected from 2009 to 2015. The measurement benefits from the large effective volume and the good directional resolution of track-like events but has a restricted field of view to the Northern hemisphere. Main backgrounds are atmospheric neutrinos and atmospheric muons produced by cosmic ray interactions in Earth's atmosphere. By selecting well reconstructed muon tracks originating from and below the horizon, atmospheric muons can be rejected efficiently. This has resulted in a high-statistics neutrino sample of about 350,000 neutrino events with a purity of $99.7 \%$. Most events are atmospheric neutrinos originating from pion and kaon decays (conventional atmospheric). A sub-dominant contribution of events from the decay of charmed mesons (prompt atmospheric) is expected. The sensitive energy range above which an extraterrestrial neutrino flux can be detected is about $200 \mathrm{TeV}$. This is larger than for starting event analysis [6], because of a larger background flux of atmospheric muon neutrino and a poorer energy resolution for uncontained through-going muon events. An evident flux of extraterrestrial neutrinos extending up to several PeV has been observed, including the highest-energy neutrino reported to date, with a median inferred neutrino energy of $7.8 \mathrm{PeV}$. The flux is well described by an isotropic, unbroken power law $E^{-\gamma}$.

Here, we update this analysis to eight years of collected data up to May $10^{\text {th }} 2017$. This corresponds to a sample of almost a half million neutrino events including almost 1000 extraterrestrial neutrinos as estimated by our best fit.

\section{The IceCube Neutrino Observatory}

The IceCube Neutrino Observatory is a cubic-kilometer sized Cherenkov detector embedded in the Antarctic ice at the South Pole [1]. It detects neutrinos by observing Cherenkov radiation emitted by charged secondary particles that are created by neutrino interactions in the ice. A total of 5160 optical sensors instrument 86 cable strings at depths between $1450 \mathrm{~m}$ and $2450 \mathrm{~m}$ beneath the surface resulting in an active volume of about one cubic kilometer.

IceCube was completed in December 2010, but it had already been taking data in the years before with the partially completed detector. The analysis presented here uses data from May 2009 to May 2017, taken with the 59-string configuration in the first year, the 79-string configuration in the second year and the 86-string configuration of the completed detector afterwards. The event 

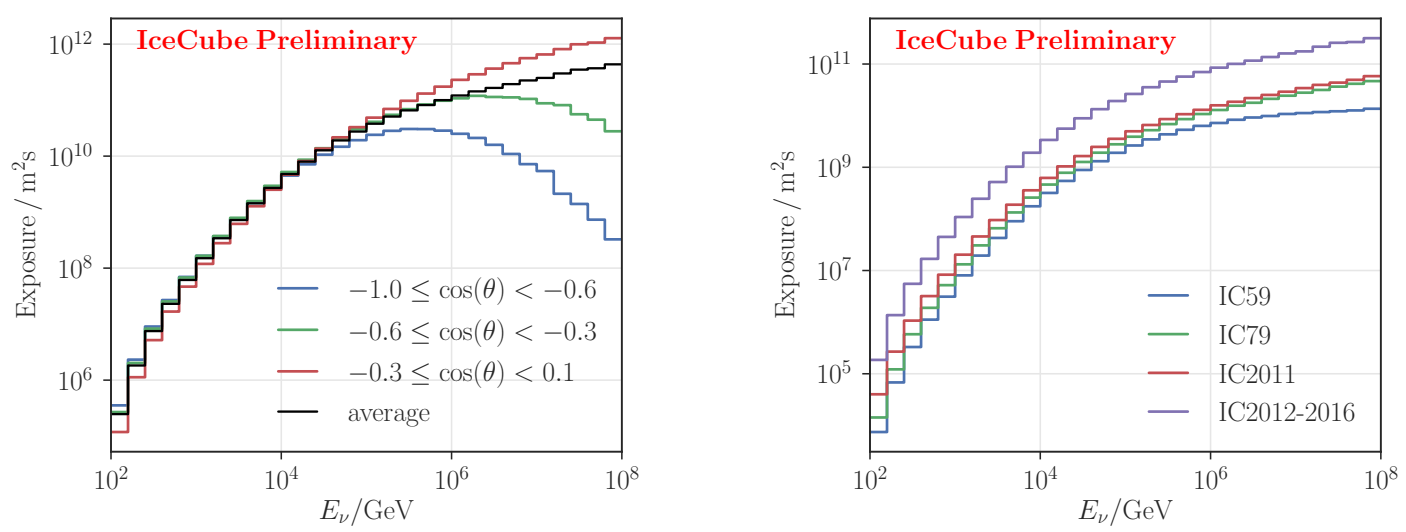

Figure 1 Exposure of the analysis, summed for neutrinos and anti-neutrinos. Left: Averaged exposure for different zenith ranges. Right: Exposure integrated over the field of view for the different detector configurations.

selection for the newly added data is unchanged with respect to [5]. The updated total integrated exposure of the analysis is shown in figure 1.

\section{Analysis Method}

\subsection{Flux Models}

We include the contribution of three sources of neutrinos in this analysis: Conventional atmospheric neutrinos from the decay of pions and kaons, prompt atmospheric neutrinos from the decay of charmed mesons, and isotropic diffuse astrophysical neutrinos. The conventional neutrino flux is modeled by the prediction of [7], modified to include the effect of different cosmic ray flux models $[8,9]$ and the cosmic-ray knee [10]. The prompt neutrino flux is modeled by the ERS model [11] with a free normalization. The astrophysical neutrino flux is modeled as an isotropic power-law flux:

$$
\frac{d \Phi}{d E}=\Phi_{0} \cdot\left(\frac{E_{V}}{100 \mathrm{TeV}}\right)^{-\gamma_{a s t r o}}
$$

\subsection{Likelihood Description}

The analysis is based on comparing the expected flux contributions obtained from MonteCarlo simulation to experimental data. Thus, the data are binned in two observables: estimated muon energy and reconstructed muon zenith angle. The expectation in each bin of the resulting histograms can be modeled by a modified Poisson likelihood $L_{i}$, which takes into account the limited statistics of the Monte-Carlo simulation [12] as described in [5].

The bin-wise expectation $\mu_{i}$ is defined as:

$$
\mu_{i}(\theta, \xi)=\mu_{i}^{\text {conv. }}(\xi)+\mu_{i}^{\text {prompt }}\left(\Phi_{\text {prompt }}, \xi\right)+\mu_{i}^{\text {astro }}\left(\Phi_{\text {astro }}, \gamma_{\text {astro }}, \xi_{\text {det. }}\right)
$$

where $\theta$ denotes the signal flux parameters $\Phi_{\text {astro }}, \gamma_{\text {astro }}, \Phi_{\text {prompt }}$ and $\xi=\left\{\xi_{\text {theo. }}, \xi_{\text {det. }}\right\}$ denotes the nuisance parameters. The nuisance parameters incorporate systematic uncertainties into the 
likelihood function. Systematic uncertainties include uncertainties in theoretical model predictions $\left(\xi_{\text {theo. }}\right)$ and detector effects $\left(\xi_{\text {det. }}\right)$ and are parametrized continuously. A detailed description of the uncertainties and their technical implementation can be found in [5]. The total likelihood is obtained as $L=\prod_{i} L_{i}$. The best-fit signal and nuisance parameters are obtained by maximizing the total likelihood function. The parameter uncertainties are obtained using the profile-likelihood technique and applying Wilks' theorem [13]. The applicability of Wilks' theorem has been verified using ensemble tests.

\subsection{Parametric Unfolding}

The observed neutrino energy spectrum can be unfolded from the observed experimental events using the posterior probability density function (PDF) $P\left(E_{v} \mid E_{\text {reco }}^{i}\right)$, where $E_{\text {reco }}^{i}$ is the muon energy proxy of event $i$. The posterior PDF is constructed by inverting the resolution functions $P\left(E_{\text {reco }}^{i} \mid E_{v}\right)$ as described in [5]. This procedure, however, depends on the prior assumed for the neutrino energy spectrum, for which we use the best-fit value of this analysis.

\section{Results}

\subsection{Updated Fit Results}

The best fit to the full data-set results in an astrophysical powerlaw flux is given as:

$$
\frac{d \Phi_{v+\bar{v}}}{d E}=\left(1.01 \pm_{0.23}^{0.26}\right)\left(\frac{E}{100 \mathrm{TeV}}\right)^{-2.19 \pm 0.10} \cdot 10^{-18} \mathrm{GeV}^{-1} \mathrm{~cm}^{-2} s^{-1} s r^{-1}
$$

This result is consistent with the previous result, that was based on six years of data but improves the accuracy. The significance of the astrophysical flux with respect to the atmospheric only hypothesis is increased from $5.6 \sigma$ to $6.7 \sigma$. The central range of neutrino energies that contribute $90 \%$ to the total observed likelihood ratio between the best-fit and the conventional atmosphericonly hypothesis in the experimental data is $119 \mathrm{TeV}$ to $4.8 \mathrm{PeV}$.
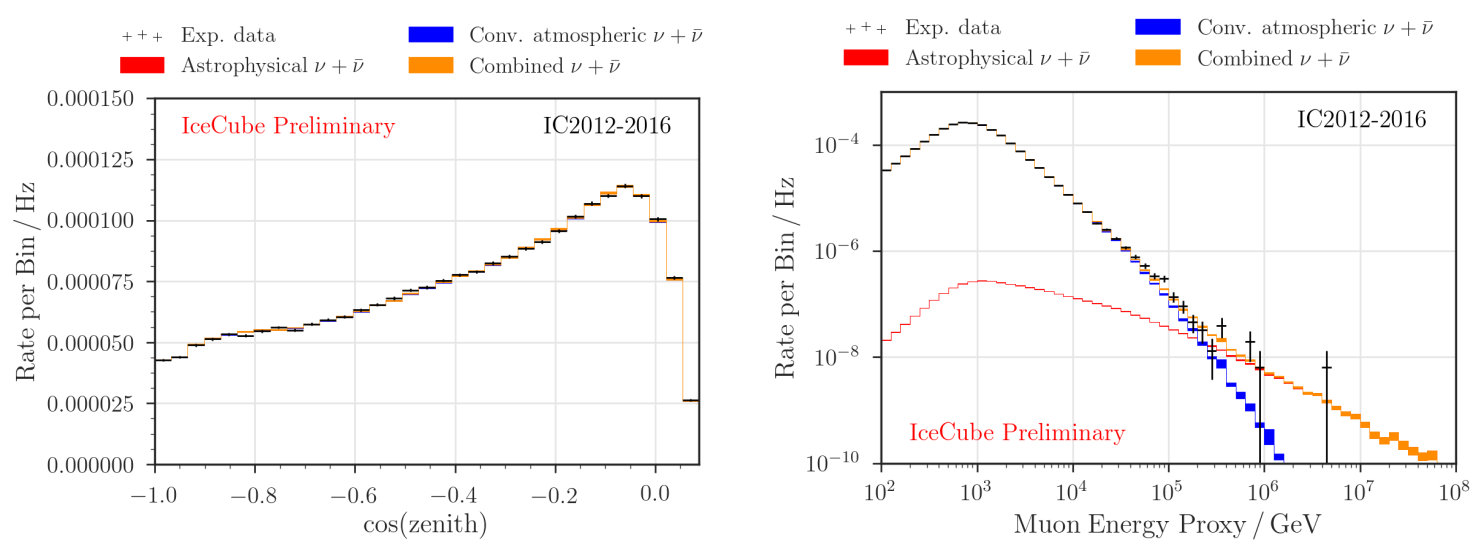

Figure 2 Fitted data distributions for the 2012-2017 detector configuration. Left: Right Projection of zenith. Projection of energy. 
Figure 2 shows the comparison of the global best fit with the observation for the most recent detector configuration with two years of added data. The fit results in a good description of the full data-set. No regions of systematic pulls are observed. Visual inspection and other cross checks of these events revealed no indication of any time dependent detector effects. Also the comparison of the earlier detector configurations (2009-2010) remains almost unchanged with respect to [5].
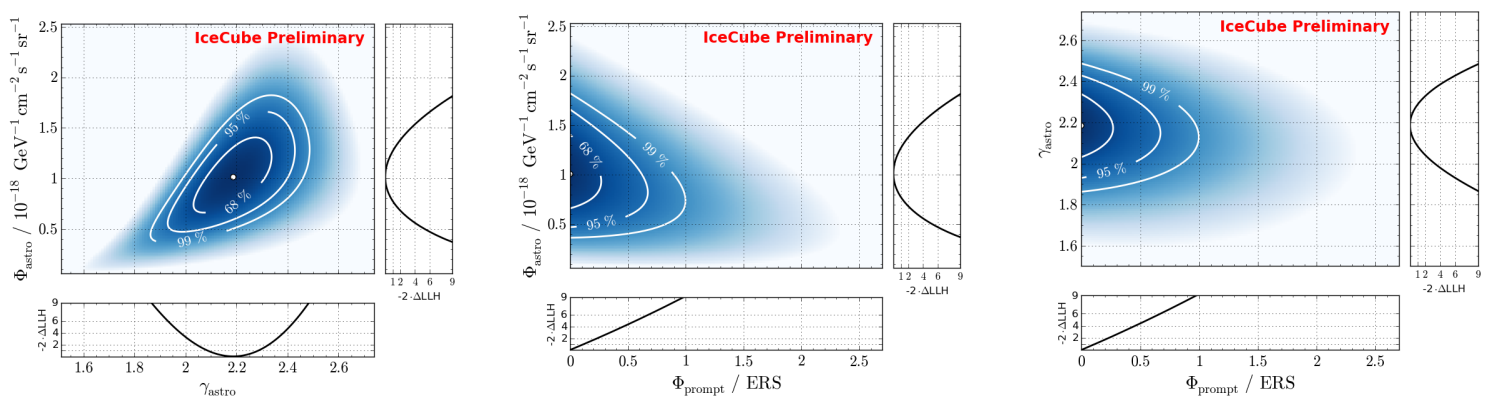

Figure 3 Scans of the profile likelihood for the signal parameters. Note that for each scan point, all other parameters are optimized. Left: Astrophysical normalization versus spectral index. Middle: Astrophysical normalization versus prompt normalization. Right: Astrophysical spectral index versus prompt normalization.

The two-dimensional contours of the profile likelihood as a function of the signal parameters are shown in figure 3 . The fitted astrophysical flux normalization is correlated with the astrophysical spectral index, whereas the prompt normalization shows only little correlation to the individual astrophysical flux parameters. Hence, an astrophysical flux with these properties would remain necessary to describe the data even for larger prompt normalizations than the current limit. However, as the prompt flux is sub-dominant to the astrophysical flux, deviations from a pure power-law (as assumed here) would result in large uncertainties for the observation of prompt neutrinos. In particular, the contour lines should not be interpreted as robust limit on the ERS flux.

\subsection{Astrophysical Flux}

The best-fit flux-models and knowledge about the relation between muon energy proxy and true neutrino energy that is derived from simulations can be used to unfold the neutrino energy distribution, as described above. Based on the per-event probability density function $P\left(E_{v} \mid E_{i}^{\text {reco }}\right)$ the median neutrino energy for each event is calculated. Figure 4 (left) shows the distribution of the median neutrino energies for the eight year sample. A clear excess above approximately $100 \mathrm{TeV}$ in neutrino energy is visible and is not compatible with the atmospheric background expectation. Although only a single event with an energy deposition greater than a PeV has been observed, we can estimate from our fit and from the relation between muon energy loss and energy of the parent neutrino that most likely several neutrinos with energies above a $\mathrm{PeV}$ are contained in the sample. The updated best-fit astrophysical flux is shown in figure 4 (right) in comparison with the measurements of high-energy starting events [6].

\subsection{Test for a Spectral Cutoff}

In addition to a fit of the baseline astrophysical flux model, we have performed a hypothesis 

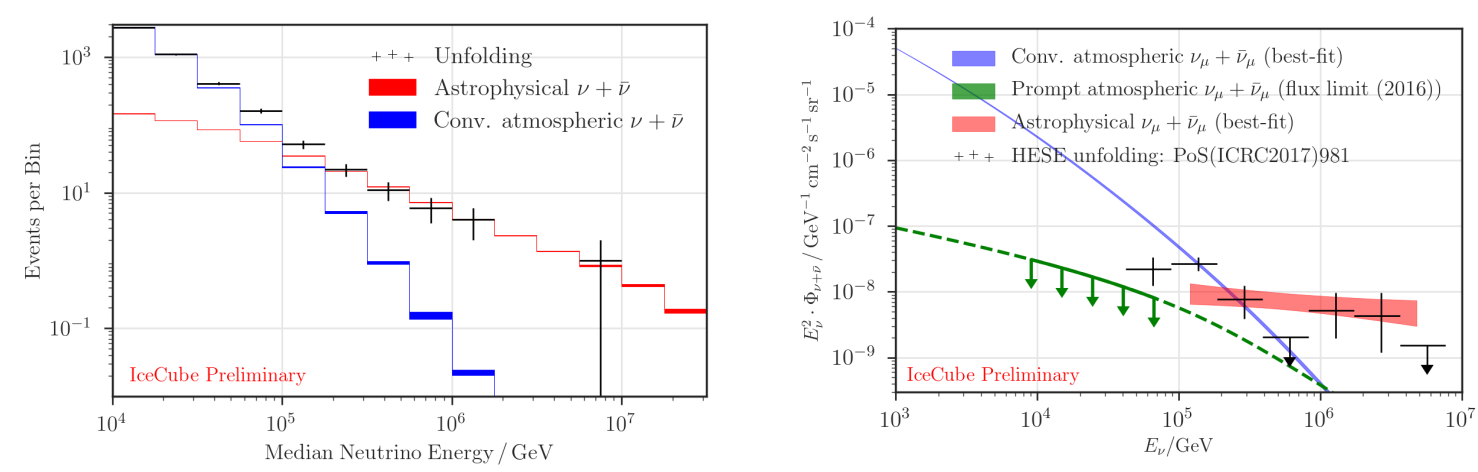

Figure 4 Measured astrophysical flux. Left: Unfolded neutrino energy spectrum in comparison to the best-fit fluxes. Right: Uncertainty range of the observed astro-physical per-flavor flux in comparison with the best fit atmospheric background and the results from the starting event analysis [6].

test for a spectral cutoff implemented as exponential factor in the flux model:

$$
\frac{d \Phi_{v+\bar{v}}}{d E}=\exp \left(-\frac{E}{E_{\text {cut }}}\right) \cdot \Phi_{\text {astro }} \cdot\left(\frac{E}{100 \mathrm{TeV}}\right)^{-\gamma_{\text {astro }}} \cdot 10^{-18} \mathrm{GeV}^{-1} \mathrm{~cm}^{-2} \mathrm{~s}^{-1} \mathrm{sr}^{-1} .
$$

The cutoff energy $E_{\text {cut }}$ is found to be strongly degenerate with a softer spectral index $\gamma$, and both parameters cannot be fitted concurrently. Therefore, the test is performed for two distinct assumptions of the astrophysical flux parameters: $(A)$ The best fit hypothesis with $\gamma_{a s t r o}=2.19$ and $(B)$ a benchmark model with $\gamma_{\text {astro }}=2.0$. All other parameters are free in the fit.
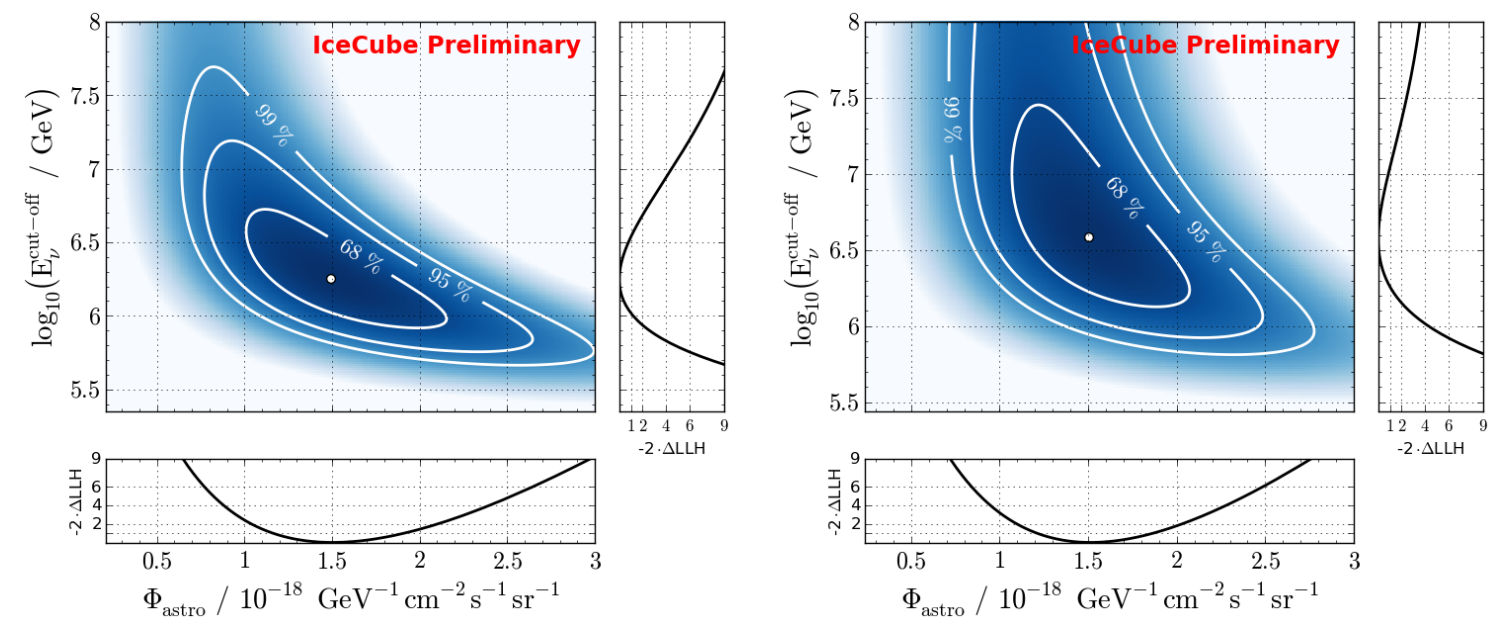

Figure 5 Likelihood scan for the hypothesis test of a spectral cut-off. Left $\gamma_{\text {astro }}=2.0$. Right: $\gamma_{\text {astro }}=2.19$

The results of both fits are shown in figure 5 as $2 \mathrm{D}$ profile likelihood scans in $E_{\text {cut }}$ and $\Phi_{\text {astro. }}$. For our best-fit spectrum $(A)$ a cut-off is not significant. However, for an index harder than preferred by our measurement a cut-off would be required. For the benchmark hypothesis $(B)$ of a hard 
Table 1 Summary of the highest energy events (above $200 \mathrm{TeV}$ estimated muon energy) in addition to the events reported in [5]. The signalness is defined as the ratio of the astrophysical expectation over the sum of the atmospheric and astrophysical expectations for a given energy proxy and the best-fit spectrum. The angular errors are statistical and do not include systematic uncertainties.

\begin{tabular}{llrcrrrr}
\hline ID & MJD & Signalness & Energy Proxy (TeV) & Decl. (deg) & 90\% C.L. & R.A. (deg) & 90\% C.L. \\
\hline 30 & 57217.9 & 0.61 & 300 & 26.1 & ${ }_{-1.85}^{+1.68}$ & 325.5 & ${ }_{-1.46}^{+1.77}$ \\
31 & 57246.8 & 0.69 & 380 & 6.0 & ${ }_{-0.34}^{+0.48}$ & 328.4 & ${ }_{-0.75}^{+0.59}$ \\
32 & 57269.8 & 0.51 & 220 & 28.0 & ${ }_{-0.47}^{+0.47}$ & 134.0 & ${ }_{-0.58}^{+0.39}$ \\
33 & 57312.7 & 0.52 & 230 & 19.9 & ${ }_{-2.21}^{+2.82}$ & 197.6 & ${ }_{-2.09}^{+2.46}$ \\
34 & 57340.9 & 0.86 & 740 & 12.6 & ${ }_{-0.58}^{+0.61}$ & 76.3 & ${ }_{-0.74}^{+0.75}$ \\
35 & 57478.6 & 0.69 & 380 & 15.6 & ${ }_{-0.60}^{+0.53}$ & 15.6 & ${ }_{-0.58}^{+0.45}$ \\
$36^{\dagger}$ & 57672.1 & 0.64 & 330 & 26.6 & - & 9.7 & - \\
\hline
\end{tabular}

${ }^{\dagger}$ Based on preliminary reconstruction methods

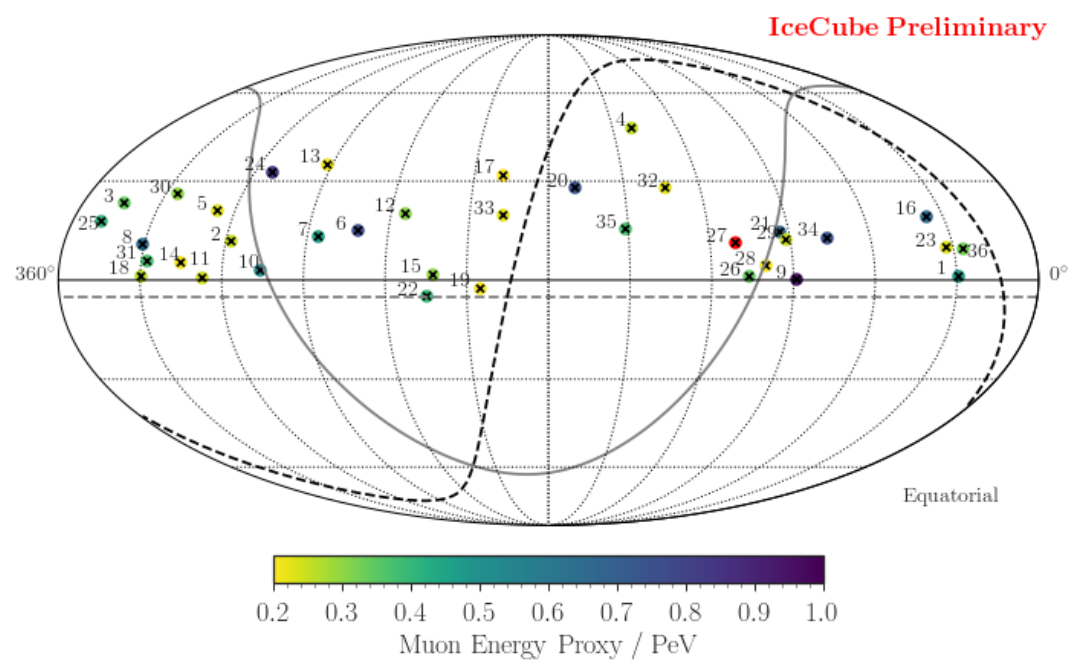

Figure 6 Reconstructed arrival directions of observed events with estimated muon energies above $200 \mathrm{TeV}$. The color indicates the energy. The number refers to table 1 and the events reported in [5]. The solid gray line indicates the galactic plane and the dashed black line the supergalactic plane.

spectrum $\left(\gamma_{\text {astro }}=2\right)$, a cutoff at $\log _{10}\left(E_{V}\right)=6.25$ is preferred at the $4.1 \sigma$ level compared to the benchmark hypothesis without cutoff.

\subsection{High Energy Events}

Seven new events with estimated muon energies above $200 \mathrm{TeV}$ have been found in the new data. Table 1 shows a summary of the event properties.

The arrival directions of these events are shown in figure 6. Similar to the previous result, no obvious correlation with astrophysical sources has been found. Also, the directions of the new events are not correlated with the directions of previous events. 


\section{Conclusions and Outlook}

We have presented the update of the analysis of up-going muon neutrinos, now covering eight years of IceCube operation, including data up to May 2017. The significance of the presence of an isotropic astrophysical muon neutrino flux with respect to a purely atmospheric origin is increased to $6.7 \sigma$. Despite of not having observed new events of very high energy depositions, the measured flux parameters $\phi_{\text {astro }}=1.01 \gamma_{\text {astro }}=2.19$ are consistent with the previously reported values.

In the previous analysis [5] we have reported an approximately 3 sigma tension in the measured astrophysical spectrum in comparison to a global fit of other IceCube results if a single unbroken power-law is assumed. With the new data reported here this tension remains at the same level. However, this tension is reduced to about 2 sigma, when compared with new data for cascades [14] and starting events [6]. Therefore the possible existence of a spectral feature remains an open question that will be particularly addressed by an update of the global fit [15]. This analysis is also able to constrain the prompt atmospheric neutrino flux. Similar to the previous result [5], no indications of the existence of a prompt flux are observed and limits are expected to further improve. Because the strong astrophysical flux is a background for this search the uncertainties due to the astrophysical flux are difficult to quantify. These studies are still ongoing, and a limit will be presented later.

\section{References}

[1] IceCube Collaboration, M. G. Aartsen et al., JINST 12 (2017) P03012.

[2] IceCube Collaboration, M. G. Aartsen et al., Science 342 (2013) 1242856.

[3] IceCube Collaboration, M. G. Aartsen et al., Phys. Rev. Lett. 113 (2014) 101101.

[4] IceCube Collaboration, M. G. Aartsen et al., Phys. Rev. Lett. 115 (2015) 081102.

[5] IceCube Collaboration, M. G. Aartsen et al., Astrophys. J. 833 (2016) 3.

[6] IceCube Collaboration, PoS ( ICRC2017) 981 (these proceedings).

[7] M. Honda et al., Phys. Rev. D75 (2007) 043006.

[8] J. R. Hoerandel, Astropart. Phys. 19 (2003) 193-220.

[9] T. K. Gaisser, T. Stanev, and S. Tilav, Front. Phys. 8 (2013) 748-758.

[10] A. Schukraft. PhD thesis, RWTH Aachen University, 2013.

[11] R. Enberg, M. H. Reno, and I. Sarcevic, Phys. Rev. D78 (2008) 043005.

[12] D. Chirkin, arXiv:1304.0735 [astro-ph.IM] (2013).

[13] S. S. Wilks, Ann. Math. Statist. 9 (1938) 60-62.

[14] IceCube Collaboration, POS ( ICRC2017) 968 (these proceedings).

[15] IceCube Collaboration, POS ( ICRC2017) 976 (these proceedings). 